Article

\title{
Analysis of Precise Orbit Predictions for a HY-2A Satellite with Three Atmospheric Density Models Based on Dynamic Method
}

\author{
Qiaoli Kong ${ }^{1,2, *}$, Fan Gao ${ }^{3,4, *}$, Jinyun Guo ${ }^{1,2}$, Litao Han ${ }^{1}$, Linggang Zhang ${ }^{1}$ and Yi Shen ${ }^{1}$ \\ 1 College of Geomatics, Shandong University of Science and Technology, Qingdao 266590, China; \\ sdk990359@sdust.edu.cn (J.G.); sdk992889@sdust.edu.cn (L.H.); sdk993850@sdust.edu.cn (L.Z.); \\ yishen1988@126.com (Y.S.) \\ 2 State Key Laboratory of Mining Disaster Prevention and Control Co-founded by Shandong Province and \\ Ministry of Science and Technology, Shandong University of Science and Technology, Qingdao 266590, China \\ 3 Institute of Space Science, Shandong University, Weihai 264209, China \\ 4 Institute of Geodesy and Geophysics, Chinese Academy of Sciences, Wuhan 430077, China \\ * Correspondence: qiaolikong@sdust.edu.cn (Q.K.); gaofan@sdu.edu.cn (F.G.); Tel.: +86-15192700186 (Q.K.); \\ $+86-13659805560$ (F.G.)
}

Received: 19 November 2018; Accepted: 25 December 2018; Published: 27 December 2018

\begin{abstract}
HY-2A (Haiyang 2A) is the first altimetry satellite in China, and it was designed to be in a repeated ground track orbit to achieve the mission targets. Maneuvers are necessary to keep the satellite on the designed orbit according to the dynamic precise orbital prediction. Atmospheric density models are essential for predicting the low Earth orbit (LEO) satellites, such as HY-2A. Nevertheless, it is a complex process to determine the optimal atmospheric density model for orbit prediction. In this paper, short-term and long-term orbit predictions based on the dynamic method using three different atmospheric density models are tested. Detailed comparisons and evaluation of the accuracy of the predicted results are performed. Furthermore, to assess the results for the ground tracking of the satellite, the interpolation method especially for a spherical surface is introduced. The results show that among the three models, the Jacchia 1971 model is in the closest agreement with Multi-Mission Ground Segment for Altimetry precise positioning and Orbitography (SSALTO) precise orbits. The root-mean-squares (RMSs) of radial orbit differences between the predicted and precise orbits are $0.016 \mathrm{~m}, 0.091 \mathrm{~m}, 0.176 \mathrm{~m}, 0.573 \mathrm{~m}$, and $1.421 \mathrm{~m}$ for predicted 1-h, 12-h, 1-day, 3-day, and 7-day arcs, respectively.
\end{abstract}

Keywords: HY-2A; obit prediction; atmospheric density models; dynamic method

\section{Introduction}

The space environment includes solar radiation, the Earth's neutral atmosphere, the regional ionosphere, the Earth's magnetic field, etc. During the travel of low Earth orbit (LEO) satellites in space, several kinds of space environment factors affect the trajectory of these missions, and atmospheric drag deduced from atmospheric density is a significant factor. These missions are loaded with observation equipment, and the observation data can be used in many fields such as meteorology, resource exploration, environmental monitoring, and space weather monitoring [1]. The precise prediction of the satellite orbit can guarantee the accuracy of the obtained observations and the validity of the data analysis. At the same time, the high-precision satellite orbit prediction is convenient to predict the satellite ground orbital interval of the repetitive cycle in advance, which will help to evaluate the environmental changes in the same region of the Earth and provide important guidance for the orbital maneuver of satellites. Therefore, precise orbit prediction and ground trajectory distance control are 
becoming more and more important and deserve special attention, especially for trajectory design and coverage assessment of altimetry satellites [2,3]. On the other hand, the orbital design of LEO navigation satellites has been put on the agenda, and precise orbit prediction will provide an important guiding role for the orbit design of such satellites.

Some research about orbit prediction for GNSS (Global Navigation Satellite System) satellites has been carried out by several authors. Mur et al. [4] performed daily orbit prediction for global positioning system (GPS) satellites, and the inter-comparison of rapid orbits shows that single-day root-mean-square (RMS) can reach $137.8 \mathrm{~cm}$. Romay and Lainez [5] focused on the generation of short-term and long-term orbit and time predictions for feeding A-GNSS, and the $14.5 \mathrm{~m}$ target (RMS) for the combined orbit and clock contribution has been achieved for most of the considered satellites for 15-day-long prediction intervals. Polle et al. [6] carried out the research of orbit/SRP (solar radiation pressure) modeling for long-term prediction for GPS and Galileo orbit to improve prediction accuracy. The above research is focused on GNSS with an altitude of about 20,000 km without the effect of atmospheric drag.

Recently, some concerns have been put into the orbit prediction of LEOs. Wang et al. [7] used the dynamic fitting method to predict the HY-2A (Haiyang 2A) orbit, and the three-dimensional position accuracy is better than $1 \mathrm{~m}$ by using a $12 \mathrm{~h}$ fitting orbit to forecast a $12 \mathrm{~h}$ arc based on the orbit being known within the whole time period. Zhu et al. [8] have performed satellite broadcast ephemeris prediction using the auto regressive moving average (ARMA) model combined with the sliding window model, and test results show that the predictability of orbit parameters has certain application value for satellite orbit prediction. The medium- and long-term orbit prediction based on the satellite broadcast ephemeris parameters is a new and feasible method of orbit prediction, though the accuracy is not very high. The above methods use the dynamic fitting method and the statistical and mathematical model to predict the orbits, respectively.

Atmospheric drag is the primary disturbing force on LEO satellites. Atmospheric drag makes satellite orbits smaller and rounder and plays a decisive role in the life of satellites. The error in the a priori atmospheric model contributes significantly to the rapid increase of the predicted orbit error. When the orbit is predicted for 20 days, the error in the a priori atmosphere model, if not properly corrected, can induce a semi-major axis error up to a few kilometers and an overall position error to several thousand kilometers [9]. Tang et al. [9] carried out orbit prediction with China's Tiangong-1 spacecraft at the altitude of about $340 \mathrm{~km}$ using the mean atmosphere model averaged from the US Naval Research Laboratory Mass Spectrometer and Incoherent Scatter Radar Extended Model 2001 NRLMSISE00 atmospheric density model, and several 20-day prediction tests show that semi-major axis errors are better than $700 \mathrm{~m}$ and overall position errors are better than $400 \mathrm{~km}$. Meng [1] solved the orbit differential equation based on the Adams-Cowell method using variable-step integration with the Jacchia 1971 atmospheric density model, introducing position and velocity interpolation formulas to improve efficiency for predicting high density ephemeris, and test results indicated that the position error was better than $500 \mathrm{~m}$ during a 7-day period. The above study did not compare the results of various atmospheric density models on orbit prediction, so no clear conclusion was given as to which model is suitable for satellite orbit prediction.

As a real-world case study, we consider the problem of predicting the orbit for the HY-2A satellite using three different atmospheric density models [10]. In this paper, to study the impact of different atmospheric density on orbit prediction for HY-2A at a $971 \mathrm{~km}$ altitude, Jacchia 1971 [11], Mass Spectrometer and Incoherent Scatter Model 1986 (MSIS86) [12] and Drag Temperature Model 87 (DTM87) [13] atmospheric density models will be chosen to predict the HY-2A orbit, and a full assessment of the impact of these three atmospheric density models on orbit prediction for the HY-2A satellite will be performed. The structure of this paper is arranged as follows. In Section 2, the models and strategies applied in the orbit prediction for HY-2A are described thoroughly, and an interpolation method special for spherical surface is introduced in the computation of equatorial distances. In Section 3, short-term and long-term period orbit prediction are carried out, and the 
results with three atmospheric density models are compared with the SSALTO ones. Furthermore, the equatorial distances are compared and discussed between the predicted and precise ground tracks and between predicted orbits of two successive repeating cycles. The equatorial distances between the successive ground tracks for the precise orbit of JASON-3, JASON-2, and HY-2A satellites are computed and analyzed. Finally, the results are discussed in Section 4, and the conclusions are drawn out in Section 5.

\section{Models and Strategies Employed in Orbit Prediction for HY-2A Satellite}

\subsection{Atmospheric Drag Model}

Atmospheric drag is the largest source of error in modeling the force on many of these LEO satellites. In this paper, a satellite orbit and geodetic parameter estimation software developed by Van Martin Systems, Inc (VMSI) was used to predict the HY-2A orbit, in which there are three popular density models, namely Jacchia 1971, MSIS86, and DTM87 atmospheric density models [14]. The widely accepted equation for atmospheric drag is $[15,16]$ :

$$
\Delta \ddot{r}_{d}=-\frac{1}{2} C_{D} \frac{A}{m_{s}} \rho v_{r}^{2} \hat{v}_{r}
$$

where the negative sign indicates that the acceleration is anti-parallel to the unit relative velocity vector $\left(\hat{v}_{r}\right)$, which provides the direction of the acceleration. $C_{D}$ is introduced to model the actual momentum transfer dependent upon the interaction between the satellite and the atmosphere. $A$ is the cross-sectional area of the orbiting body and is normal to the velocity vector. $m_{S}$ is the mass of the orbiting body. $v_{r}$ and $\rho$ are the velocity of the satellite and the atmospheric density, respectively.

The Jacchia 1971 model was a widely used standard [11,17]. The Jacchia model considers all the solar phenomena and adds temperature and/or density corrections to each of the phenomena within the integration of the conduction equation. For a certain phenomenon, this model uses the curve fitting method on a real spacecraft data to precisely signify actual atmospheric conditions. However, this method limits the model for a real-time application because it has no standard set of lookup tables, and the temperature and density profile must be determined by numerical integration every single time [17].

The so-called mass spectrometer and incoherent backscatter (MSIS) model was constructed using both ground-based incoherent backscatter radar measurements and satellites equipped with mass spectrometers and accelerometers. The MSIS models generally used all the data available from Jacchia models plus "Barlier data," accelerometer data, and the atmospheric decay model data. However, obtaining these data at the same time becomes difficult [16]. It contains mass spectrometer data from satellites and radar scatter data from the ground to develop the atmospheric model. The MSIS family of models has demonstrated to be accurate and applicable to some hard problems [18].

The DTM87 model was designed specifically to evaluate satellite drag $[13,17]$. The model was based on the hypothesis of an independent static diffuse equilibrium of different thermospheric constituents: $\mathrm{H}, \mathrm{He}, \mathrm{O}, \mathrm{N}_{2}$, and $\mathrm{O}_{2}$ as adopted in other previous thermospheric models [19]. The differential equation of diffuse equilibrium leads, by integration, to the concentration of each major constituent according to the altitude law.

The Jacchia 1971 model is mostly based on drag data obtained by observing the orbital motion of numerous satellites. On the other hand, satellite mass spectrometer and ground-based incoherent scatter radar data are the major data sources of MSIS86, and this model has not included drag measurements and satellite-borne accelerometer data. The significant difference between the DTM87 and the Jacchia 1971 models is the density of each atmospheric constituent $\left(\mathrm{N}_{2}, \mathrm{O}, \mathrm{He}\right)$, and in the DTM, the density is expanded in terms of spherical harmonics. 


\subsection{Interpolation Methods of Ground Track}

Since the satellite ground track distance between adjacent periods cannot exceed a certain distance tolerance, the distance difference between the trajectories at the same latitude must be calculated, and the difference between the repeated trajectories becomes a test standard to validate the orbit prediction and a guide for orbital maneuvering [2,3]. As the satellite's ground trajectory is laid out along a spherical surface, the general interpolation and fitting methods are not suitable for calculating the intersection of the ground trajectory and the Earth's equator. Therefore, we applied the interpolation method special for spherical surface to calculate the position of the intersection. The crossing nodes on equator are named ascending nodes and descending nodes. The mathematical models computing the longitudes of equatorial crossing points are listed as Equations (2) and (3), and the diagrammatic sketch is shown in Figure 1 [20,21]:

$$
\begin{gathered}
\lambda=\lambda_{P}-\frac{\varphi_{P}-\varphi_{0}}{D \cdot \cos \varphi_{0}} \\
D=\frac{\varphi_{P}-\varphi_{Q}}{\left(\lambda_{P}-\lambda_{Q}\right) \cos \varphi_{0}}
\end{gathered}
$$

where $\lambda$ is the longitudes of equatorial crossing points, $\varphi_{P}$ and $\varphi_{Q}$ are the latitudes of two adjacent points distributed on both sides of the equator according to the order of time, $\lambda_{P}$ and $\lambda_{Q}$ are longitudes of these two points, and $D$ is an intermediate variable introduced to compute the longitude for the intersection.

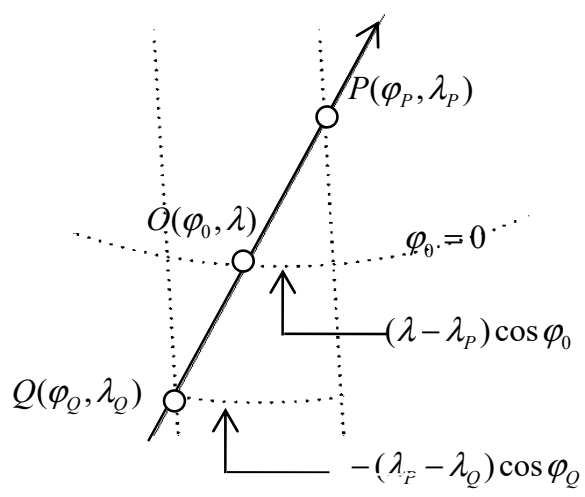

(a) Ascending arc

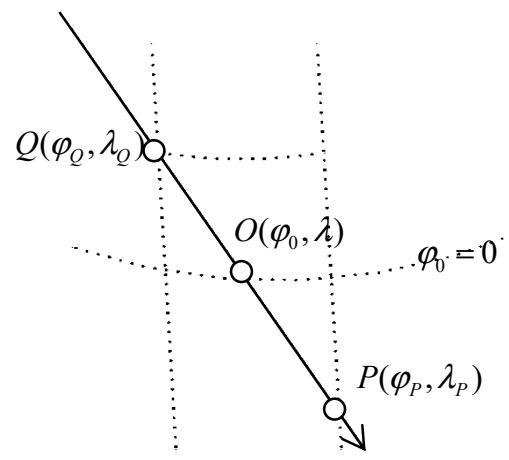

(b) Descending arc

Figure 1. The diagram for the computation of equatorial longitude of ground track: (a) ascending, and (b) descending.

\subsection{The Orbit Prediction Strategy}

VMSI software package is used to predict the HY-2A dynamic orbits in this paper. In the process of orbit prediction for HY-2A using the dynamic method, six prior independent parameters at the initial epoch are needed for the orbit integration. These six parameters can be the three-dimensional position and velocity vectors or six orbital elements. HY-2A is disturbed by the conservative forces and the non-conservative forces as this mission travels around the Earth [22]. The conservative forces include the Earth's gravitation, n-body perturbation, oceanic and solid Earth tides, etc., and the non-conservative forces include the solar and Earth radiations, atmospheric drag and relativistic effect, and other small perturbing factors. The multi-step COWELL II numerical integration was used during the process of orbit prediction for HY-2A [23], and the step size for orbit integration was $10 \mathrm{~s}$. The Doppler Orbitography and Radiopositioning Integrated by Satellite (DORIS) stations can be used to predict the observation of the tracking stations. The applied dynamic models for the HY-2A satellite are summarized in Table 1. 
Table 1. Dynamic models for precise orbit prediction of HY-2A.

\begin{tabular}{cc}
\hline Items & Description \\
\hline Coordinates of DORIS beacon stations & http:/ $/$ www.ipgp.fr/ $\{$ willis/DPOD2008/ \\
Earth gravity model & EGM2008 [24], 80 $\times 80$ \\
N-body & JPL DE403 [25] \\
Solid Earth tides & IERS2010 [26] \\
Ocean tides and ocean tide loading & FES2004 [27] \\
Relativistic effect & IERS2003 [28] \\
Solar radiation pressure & Box-Wing [29] \\
Earth albedo radiation & Knocke-Ries-Tapley [30] \\
Tropospheric model & Hopfied [31] \\
Atmospheric drag & MSIS86 [12], Jacchia 1971 [11], DTM87 [13]
\end{tabular}

HY-2A satellite has a near sun-synchronous frozen orbit. Its altitude is about $971 \mathrm{~km}$, inclination is about $99.3^{\circ}$, and the period is about $104.45 \mathrm{~min}$. Its equator crossing time on descending node is at 6:00 $\mathrm{h}$, and its repeat cycle has two phases (14 days/193 revolutions and 168 days/2315 revolutions). The HY-2A satellite has a complex shape. Table 2 gives the details of spacecraft surfaces and radiators, including the projected areas and optical characters. The solar arrays of HY-2A are unmovable, therefore the 13-plate macro-model of HY-2A is formed based on the information listed in Table 2 [32].

Table 2. The optical and infrared properties of the macro-model and the plate surfaces.

\begin{tabular}{cccccccc}
\hline \multirow{2}{*}{ Surface $\left(\mathbf{m}^{2}\right)$} & \multicolumn{2}{c}{ Normal in Satellite Reference Frame } & \multicolumn{2}{c}{ Optical Properties } & \multicolumn{2}{c}{ Infrared Properties } \\
\cline { 2 - 7 } & $\mathbf{X}$ & $\mathbf{Y}$ & $\mathbf{Z}$ & Diffuse & Emissivity & Diffuse & Emissivity \\
\hline 2.50 & 1 & & & 0.54 & 0.46 & 0.31 & 0.69 \\
2.92 & -1 & & 0.54 & 0.46 & 0.31 & 0.69 \\
5.85 & & -1 & & 0.54 & 0.46 & 0.31 & 0.69 \\
6.74 & & & 0.54 & 0.46 & 0.31 & 0.69 \\
4.93 & & & -1 & 0.54 & 0.46 & 0.31 & 0.69 \\
4.60 & & & & 0.54 & 0.46 & 0.31 & 0.69 \\
9.06 & & & & 0.06 & 0.64 & 0.16 & 0.84 \\
9.06 & & & & 0.85 & 0.15 & 0.06 & 0.94 \\
0.71 & 1 & & & 0.85 & 0.15 & 0.21 & 0.79 \\
0.60 & 1 & & & 0.73 & 0.27 & 0.13 & 0.79 \\
0.89 & & & & 0.85 & 0.15 & 0.21 & 0.79 \\
1.50 & & & 1 & 0.85 & 0.15 & 0.21 & 0.79 \\
1.80 & & & & & & & \\
\hline
\end{tabular}

\section{Tests and Results}

\subsection{Predicted Orbit and SSALTO Orbit Comparison within Short-Term and Long-Term Arc Periods}

\subsubsection{Orbital Comparison within Short-Term Arc Period}

We have predicted HY-2A orbits for 1 h, 2 h, 4 h, 8 h, 12 h, and 24 h using Jacchia 1971, MSIS86, and DTM87 atmospheric density models, separately. The SSALTO precise orbit named SSALTO or SSA orbit are released by the CNES-SSALTO/POD team in sp3 format for the missions with a DORIS receiver onboard. The accuracy of the precise orbit can reach $1.1 \mathrm{~cm}$ in the radial direction [32]. The precise orbits released by SSALTO are used as a reference for the computation and evaluation of the prediction errors, which are the orbital biases of predicted orbits from the HY-2A precise orbits in the section. The orbital biases are plotted in Figures 2-4, respectively. The statistics are listed in Tables 3-5, respectively. 

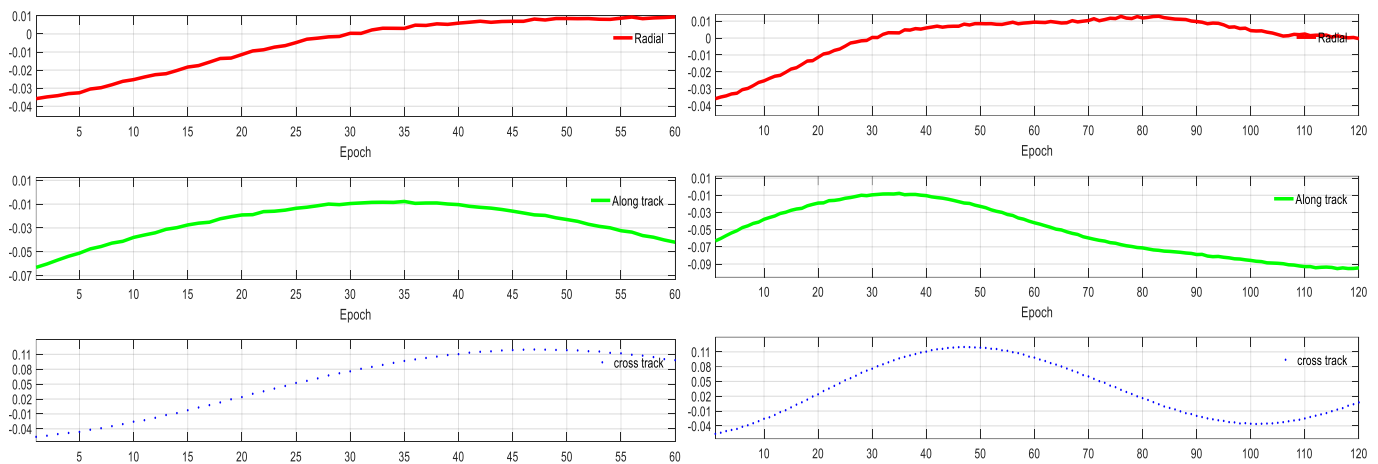

(a)

(b)

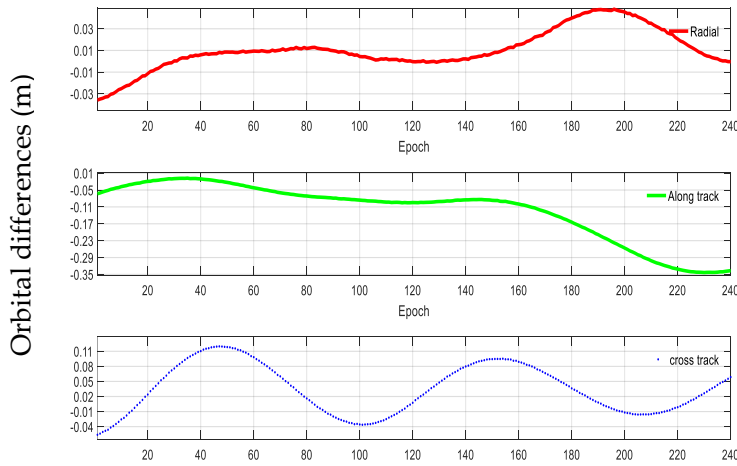

(c)
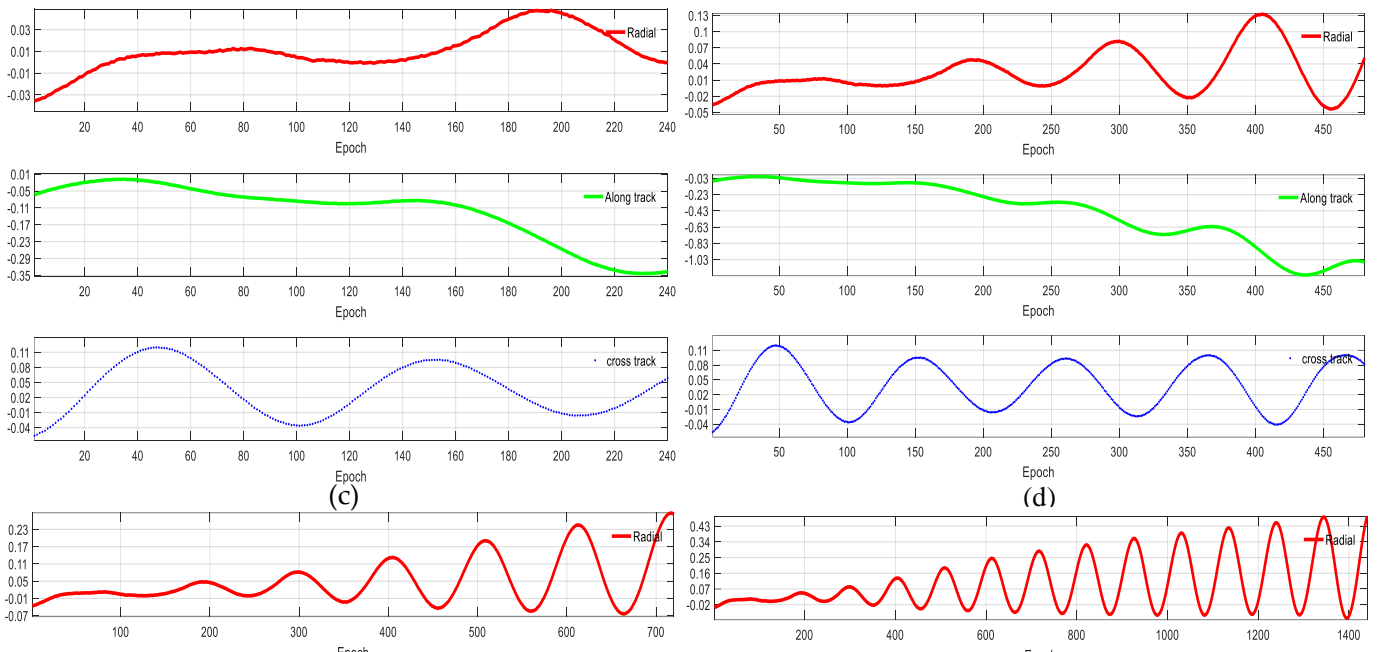

(d)
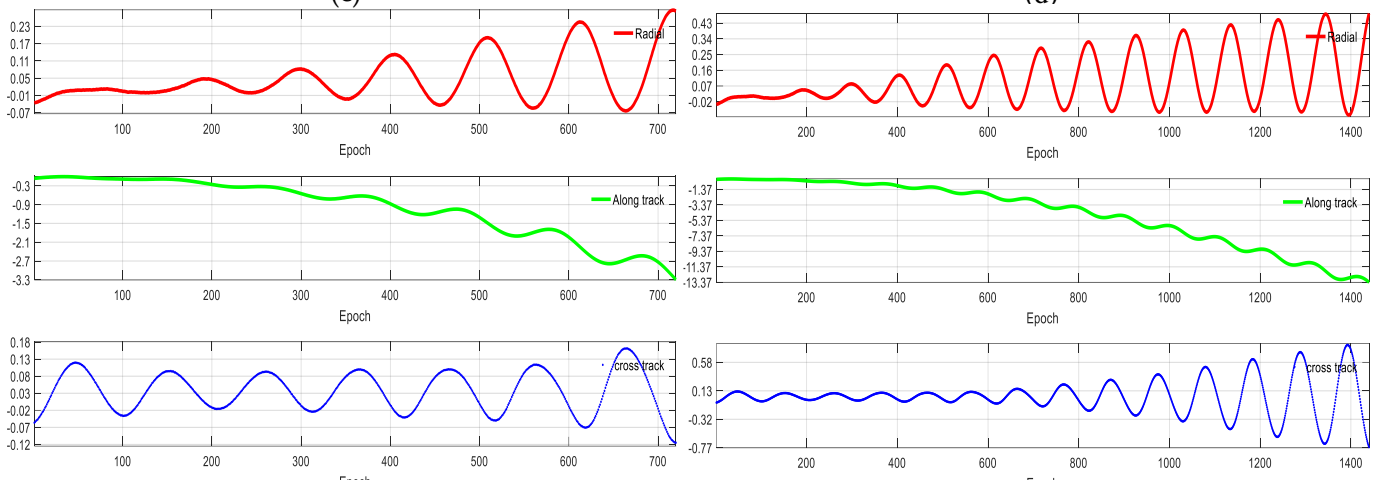

(e)



(f)

Figure 2. Differences between the predicted orbits and precise orbits within $1 \mathrm{~h}(\mathbf{a}), 2 \mathrm{~h} \mathrm{(b)}, 4 \mathrm{~h}(\mathbf{c}), 8 \mathrm{~h}$ (d), $12 \mathrm{~h}(\mathbf{e})$, and $24 \mathrm{~h}$ (f) using the Jacchia 1971 atmospheric density model.

Table 3. The statistics of orbit differences between the predicted orbits and precise orbits within $1 \mathrm{~h}$, $2 \mathrm{~h}, 4 \mathrm{~h}, 8 \mathrm{~h}, 12 \mathrm{~h}$, and $24 \mathrm{~h}$ using the Jacchia 1971 atmospheric density model (m).

\begin{tabular}{cccccccc}
\hline & \multicolumn{2}{c}{ Radial } & \multicolumn{2}{c}{ Along Track } & \multicolumn{2}{c}{ Cross Track } & 3-D \\
\cline { 2 - 8 } & (Standard Deviation) STD & RMS & STD & RMS & STD & RMS & RMS \\
\hline $1 \mathrm{~h}$ & 0.015 & 0.016 & 0.015 & 0.029 & 0.061 & 0.082 & 0.089 \\
$2 \mathrm{~h}$ & 0.013 & 0.013 & 0.030 & 0.059 & 0.057 & 0.065 & 0.089 \\
$4 \mathrm{~h}$ & 0.018 & 0.021 & 0.103 & 0.160 & 0.048 & 0.059 & 0.172 \\
$8 \mathrm{~h}$ & 0.039 & 0.045 & 0.382 & 0.579 & 0.048 & 0.062 & 0.584 \\
$12 \mathrm{~h}$ & 0.077 & 0.091 & 0.921 & 1.363 & 0.058 & 0.068 & 1.367 \\
$24 \mathrm{~h}$ & 0.144 & 0.176 & 4.101 & 6.045 & 0.262 & 0.265 & 6.054 \\
\hline
\end{tabular}

As can be seen in Figure 2 and Table 3, the disagreement among the predicted orbits using Jacchia 1971 and the precise orbits became larger and larger with the extension of the time span, and the divergence phenomenon became more and more significant. Table 3 shows that the RMSs of disagreement between the predicted orbits and precise orbits in radial direction were just $0.016 \mathrm{~m}$, 
$0.013 \mathrm{~m}, 0.021 \mathrm{~m}$, and $0.045 \mathrm{~m}$ over $1 \mathrm{~h}, 2 \mathrm{~h}, 4 \mathrm{~h}$ and $8 \mathrm{~h}$, respectively, and the ones for $12 \mathrm{~h}$ and 1 day were $0.0913 \mathrm{~m}$ and $0.176 \mathrm{~m}$, respectively. The above analysis indicated that the predicted orbits had good agreement with the precise orbits in radial direction.
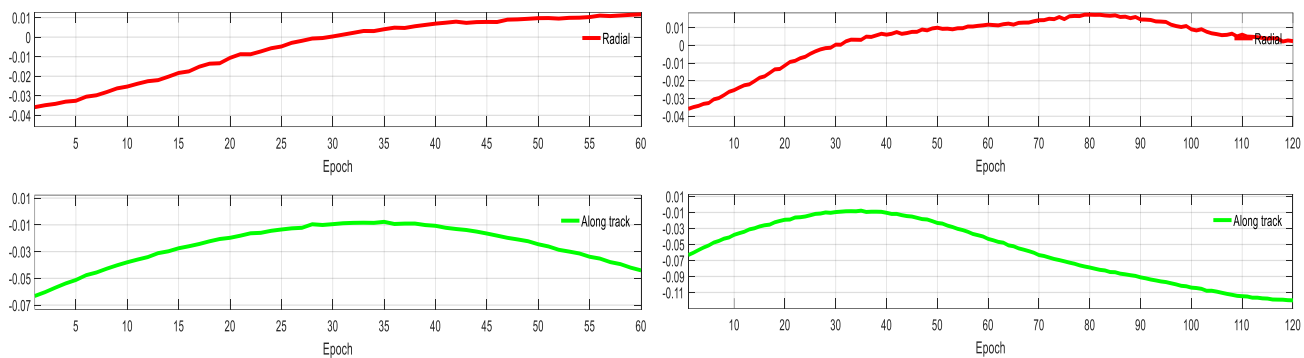

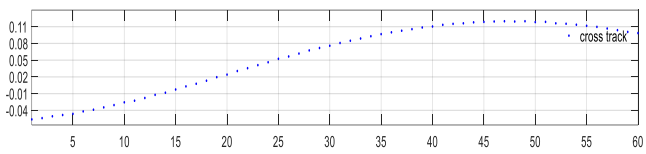

(a)
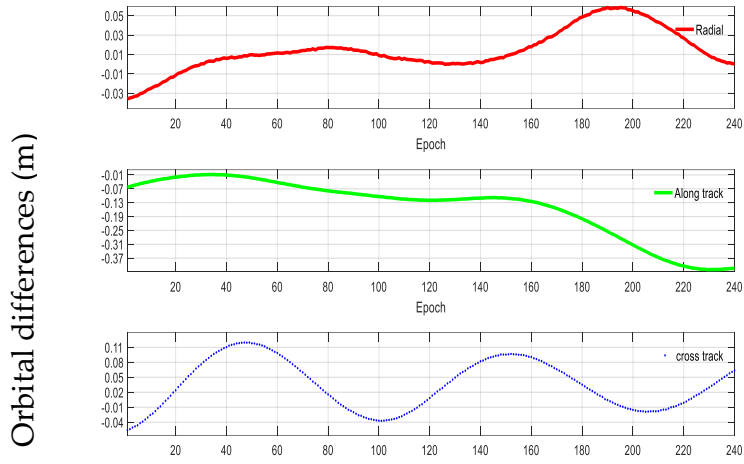

(c)
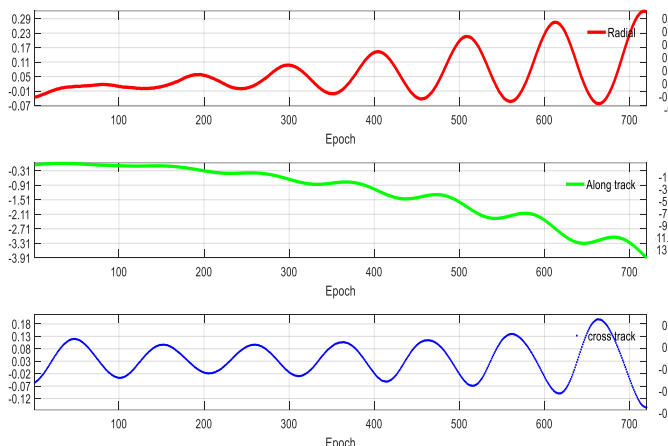

(e)

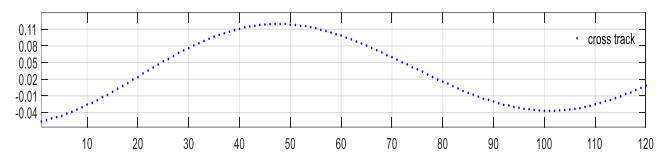

(b)
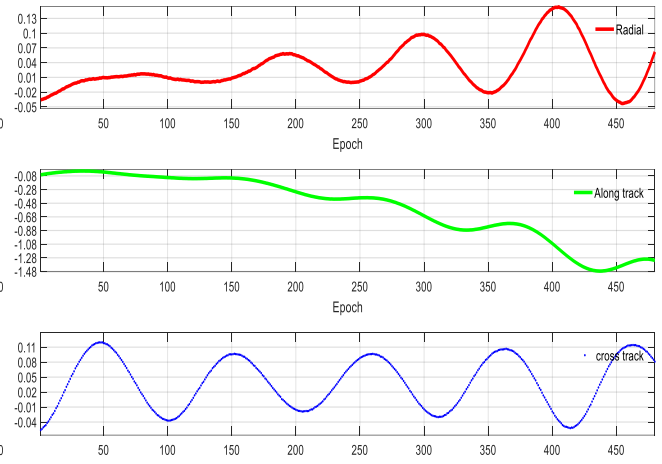

(d)
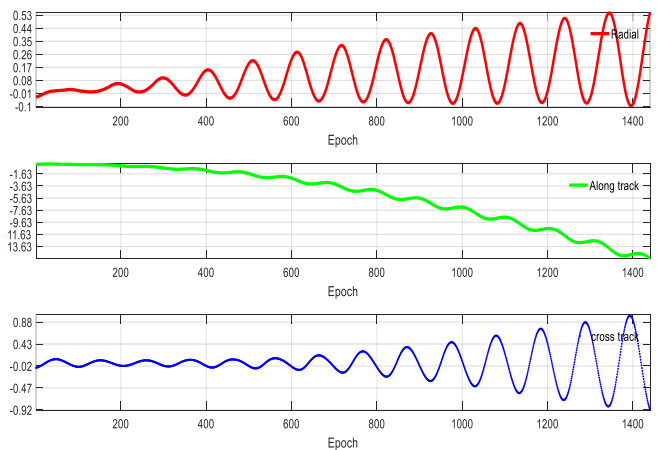

(f)

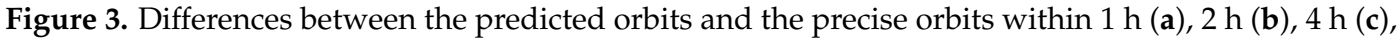
$8 \mathrm{~h}(\mathrm{~d}), 12 \mathrm{~h}(\mathbf{e})$, and $24 \mathrm{~h}(\mathbf{f})$ using the MSIS86 atmospheric density model.

Table 4. The statistics of orbit differences between the predicted orbits and the SSALTO ones within $1 \mathrm{~h}, 2 \mathrm{~h}, 4 \mathrm{~h}, 6 \mathrm{~h}, 12 \mathrm{~h}$, and $24 \mathrm{~h}$ using the MSIS86 atmospheric density model (m).

\begin{tabular}{cccccccc}
\hline & \multicolumn{2}{c}{ Radial } & \multicolumn{2}{c}{ Along Track } & \multicolumn{2}{c}{ Cross Track } & \multicolumn{2}{c}{ 3-D } \\
\cline { 2 - 8 } & STD & RMS & STD & RMS & STD & RMS & RMS \\
\hline $1 \mathrm{~h}$ & 0.015 & 0.016 & 0.015 & 0.029 & 0.061 & 0.082 & 0.089 \\
$2 \mathrm{~h}$ & 0.014 & 0.014 & 0.038 & 0.068 & 0.057 & 0.065 & 0.095 \\
$4 \mathrm{~h}$ & 0.021 & 0.025 & 0.128 & 0.195 & 0.049 & 0.060 & 0.206 \\
$8 \mathrm{~h}$ & 0.044 & 0.053 & 0.467 & 0.707 & 0.051 & 0.065 & 0.712 \\
$12 \mathrm{~h}$ & 0.086 & 0.103 & 1.102 & 1.639 & 0.069 & 0.078 & 1.644 \\
$24 \mathrm{~h}$ & 0.160 & 0.200 & 4.783 & 7.084 & 0.314 & 0.316 & 7.094 \\
\hline
\end{tabular}


As can be observed in Figure 3 and Table 4, the situation for the predicted orbits using MSIS86 had a similar phenomenon to the ones using Jacchia 1971, as seen in Figure 2. The RMSs of the disagreement in radial direction were $0.016 \mathrm{~m}, 0.014 \mathrm{~m}, 0.025 \mathrm{~m}$, and $0.053 \mathrm{~m}$ for $1 \mathrm{~h}, 2 \mathrm{~h}, 4 \mathrm{~h}$, and $8 \mathrm{~h}$, respectively, and the ones for $12 \mathrm{~h}$ and 1 day were $0.103 \mathrm{~m}$ and $0.200 \mathrm{~m}$, respectively. The results show that the predicted orbits were very close to the SSALTO orbits. From Tables 3 and 4, we can see that the values of RMS for predicted orbits using MSIS86 were somewhat larger than those using Jacchia 1971.
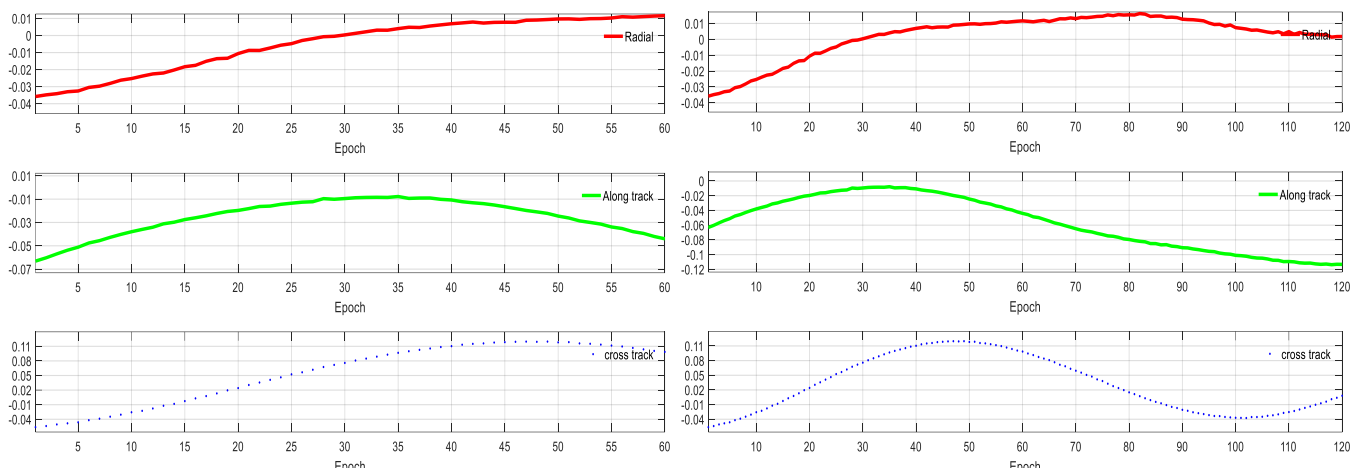

(a)

(b)
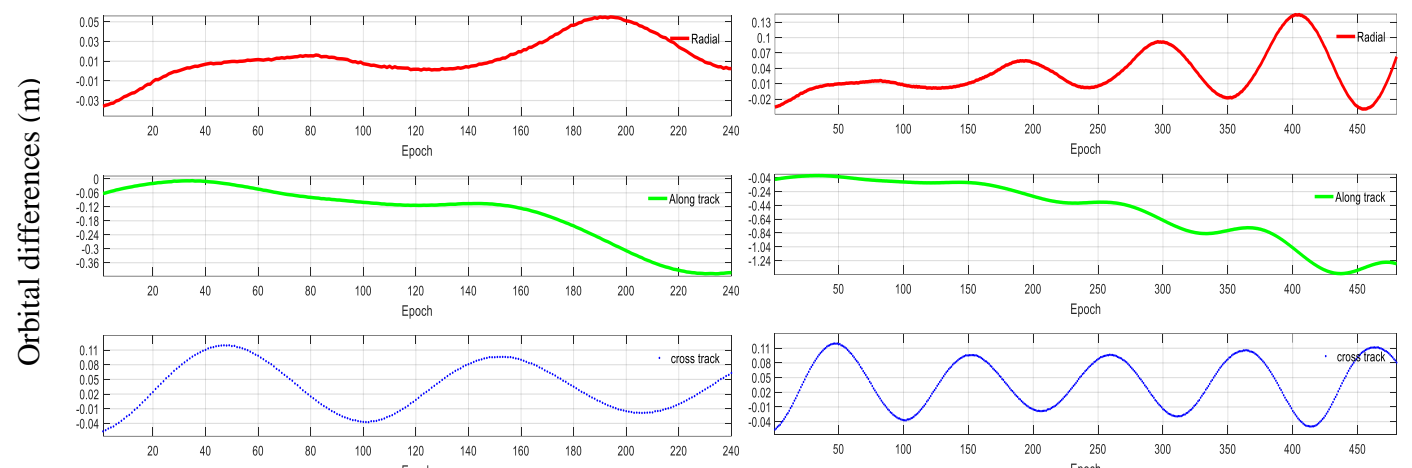

(c)

(d)


(eoch

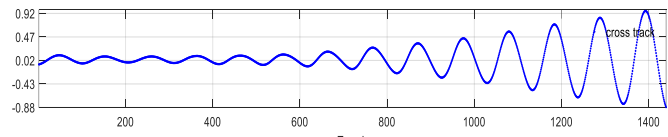

(f)

Figure 4. Differences between the predicted orbits and the precise orbits within $1 \mathrm{~h} \mathrm{(a),} 2 \mathrm{~h} \mathrm{(b),} 4 \mathrm{~h} \mathrm{(c),}$ $8 \mathrm{~h}(\mathbf{d}), 12 \mathrm{~h}(\mathbf{e})$, and $24 \mathrm{~h}(\mathbf{f})$ using the DTM87 atmospheric density model. 
Table 5. The statistics of orbit differences between the predicted orbits and the precise ones within $1 \mathrm{~h}$, $2 \mathrm{~h}, 4 \mathrm{~h}, 8 \mathrm{~h}, 12 \mathrm{~h}$, and $24 \mathrm{~h}$ using the DTM87 atmospheric density model (m).

\begin{tabular}{clllllll}
\hline & \multicolumn{2}{c}{ Radial } & \multicolumn{2}{c}{ Along Track } & \multicolumn{2}{c}{ Cross Track } & 3-D \\
\cline { 2 - 8 } & STD & RMS & STD & RMS & STD & RMS & RMS \\
\hline 1 h & 0.015 & 0.016 & 0.015 & 0.029 & 0.061 & 0.082 & 0.089 \\
$2 \mathrm{~h}$ & 0.014 & 0.014 & 0.036 & 0.067 & 0.057 & 0.065 & 0.094 \\
$4 \mathrm{~h}$ & 0.020 & 0.024 & 0.124 & 0.191 & 0.049 & 0.060 & 0.201 \\
$8 \mathrm{~h}$ & 0.042 & 0.050 & 0.454 & 0.690 & 0.051 & 0.064 & 0.695 \\
$12 \mathrm{~h}$ & 0.082 & 0.099 & 1.056 & 1.578 & 0.067 & 0.076 & 1.583 \\
$24 \mathrm{~h}$ & 0.153 & 0.191 & 4.591 & 6.800 & 0.300 & 0.302 & 6.809 \\
\hline
\end{tabular}

Figure 4 shows that the disagreement among the predicted orbits using DTM87 and the SSALTO orbits has a similar phenomenon with the results derived from the above two atmospheric densities. Table 5 shows that the RMSs of disagreement in radial direction are $0.016 \mathrm{~m}, 0.014 \mathrm{~m}, 0.024 \mathrm{~m}$, and $0.050 \mathrm{~m}$ corresponding to $1 \mathrm{~h}, 2 \mathrm{~h}, 4 \mathrm{~h}$, and $8 \mathrm{~h}$, respectively, and the ones for $12 \mathrm{~h}$ and 1 day are $0.099 \mathrm{~m}$ and $0.191 \mathrm{~m}$, respectively. The accuracy in radial direction was a little lower than that of using Jacchia 1971 and better than that when using MSIS86.

From the above analysis, we can see that all three models had good agreement with precise orbits, with the RMS even for one day all being better than $0.200 \mathrm{~m}$ compared with the precise orbits in radial component. Tables 3-5 denote that the accuracy of the predicted orbits for $1 \mathrm{~h}$ time span was somewhat lower than those for $2 \mathrm{~h}$. The main reason is that the integrator oscillates when it starts to integrate, but it becomes stable after a while. In general, the predicted orbits in three dimensions using Jacchia 1971 were better than the results using the other two models.

\subsubsection{Orbital Comparison within a Long-Term Arc Period}

In order to evaluate the effect of the orbital prediction strategy for long-term, the arc length was extended to 3 days and 7 days, and orbits fitted to the SSALTO orbits were performed using Jacchia 1971, MSIS86 and DTM87 atmospheric density models. The orbital differences between the predicted orbits and SSALTO ones using MSIS86, Jacchia 1971, and DTM87 models are plotted in Figures 5-7, respectively, and the statistics are summarized in Tables $6-8$, respectively.

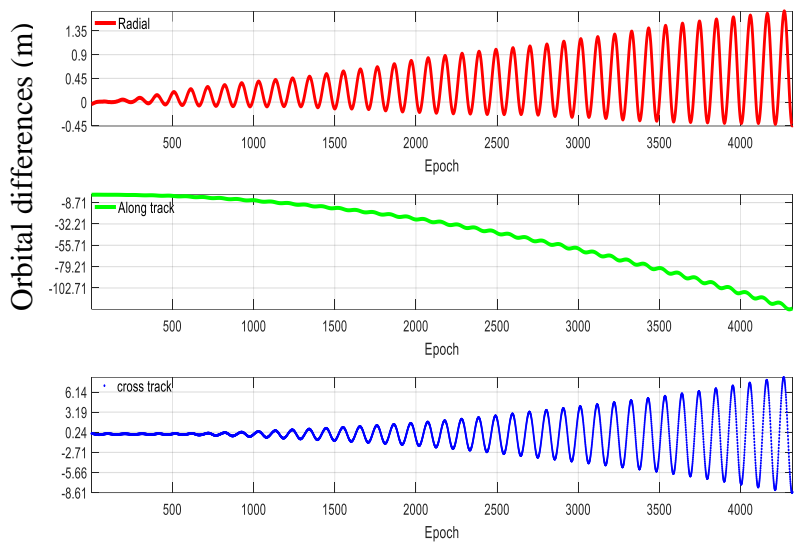

(a)
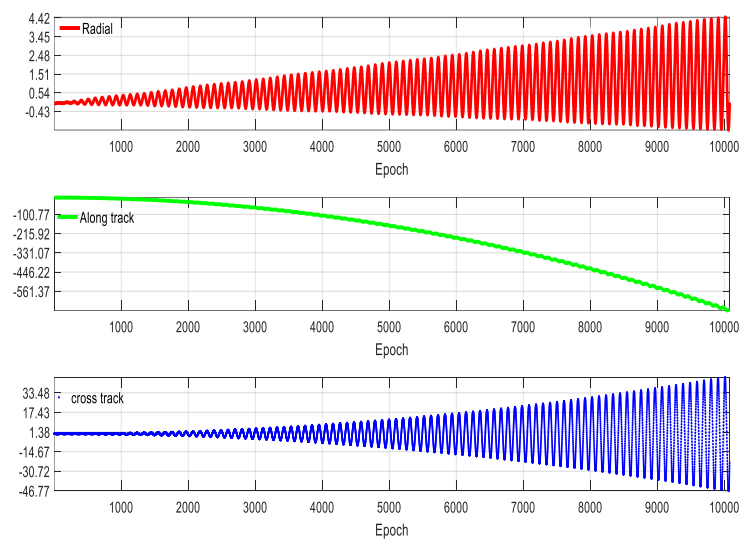

(b)

Figure 5. The orbital differences between the predicted orbits and the precise ones within 3 days (a), and 7 days (b), using the Jacchia 1971 atmospheric density model. 
Table 6. The statistics of orbital differences between the predicted ones and the SSALTO ones within 3 days and 7 days using the Jacchia 1971 atmospheric density model (m).

\begin{tabular}{lccccccc}
\hline & \multicolumn{2}{c}{ Radial } & \multicolumn{2}{c}{ Along Track } & \multicolumn{2}{c}{ Cross Track } & 3-D \\
\cline { 2 - 8 } & STD & RMS & STD & RMS & STD & RMS & RMS \\
\hline 3 days & 0.475 & 0.573 & 37.488 & 56.006 & 2.664 & 2.664 & 56.073 \\
7 days & 1.210 & 1.421 & 201.783 & 303.025 & 14.812 & 14.812 & 303.39 \\
\hline
\end{tabular}

As can be seen in Figure 5 and Table 6, consistencies between the predicted orbits with Jacchia 1971 and the precise orbits using 3-day arc were significantly better than that when using the 7-day arc in three components, and the disagreement between the predicted orbits with Jacchia 1971 and the precise orbits for 3-day arc was $0.573 \mathrm{~m}$ and the one for 7-day arc was $1.421 \mathrm{~m}$ in the radial direction.

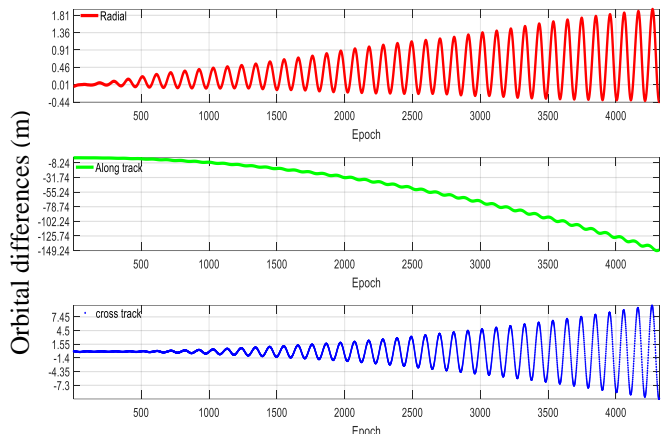

(a)
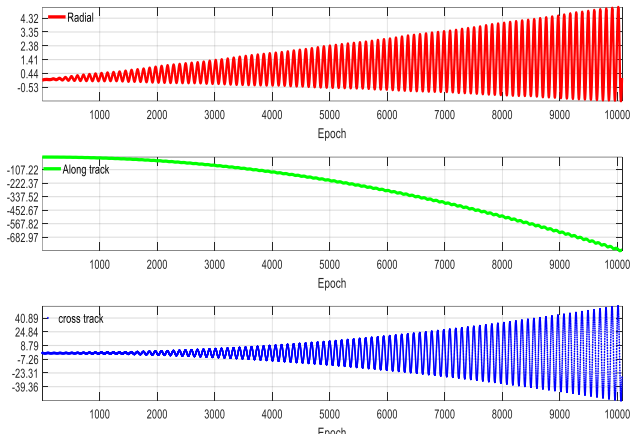

(b)

Figure 6. The orbital differences between the predicted orbits and the SSALTO ones within 3 days (a), and 7 days (b), using the MSIS86 atmospheric density model.

Table 7. The statistics of orbital differences between the predicted orbits and the precise orbits within 3 days and 7 days using the MSIS86 atmospheric density model (m).

\begin{tabular}{cccccccc}
\hline & \multicolumn{2}{c}{ Radial } & \multicolumn{2}{c}{ Along Track } & \multicolumn{2}{c}{ Cross Track } & 3-D \\
\cline { 2 - 8 } & STD & RMS & STD & RMS & STD & RMS & RMS \\
\hline 3 days & 0.532 & 0.653 & 44.304 & 66.073 & 3.171 & 3.171 & 66.152 \\
7 days & 1.361 & 1.621 & 238.858 & 358.794 & 17.622 & 17.621 & 359.23 \\
\hline
\end{tabular}

It can be observed in Figure 6 and Table 7 that agreement between the predicted orbits with MSIS86 and the SSALTO orbits using the 3-day arc was significantly better than that of using the 7-day arc in three components, and for the 3-day arc, the RMS of the inconsistency was $0.653 \mathrm{~m}$ in the radial direction and that for the 7-day arc was $1.621 \mathrm{~m}$.

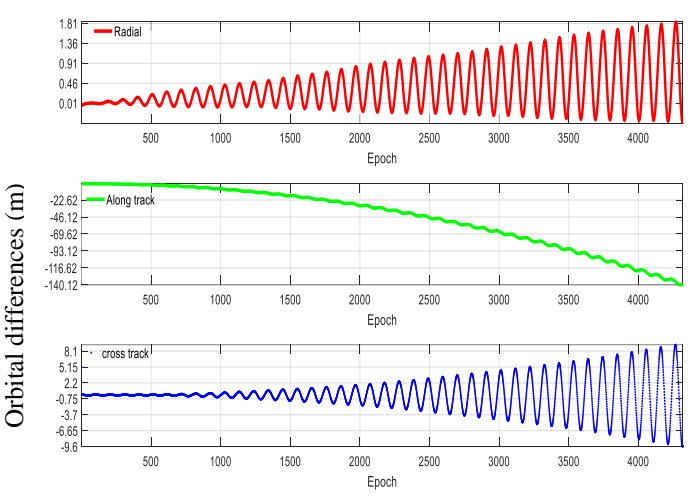

(a)
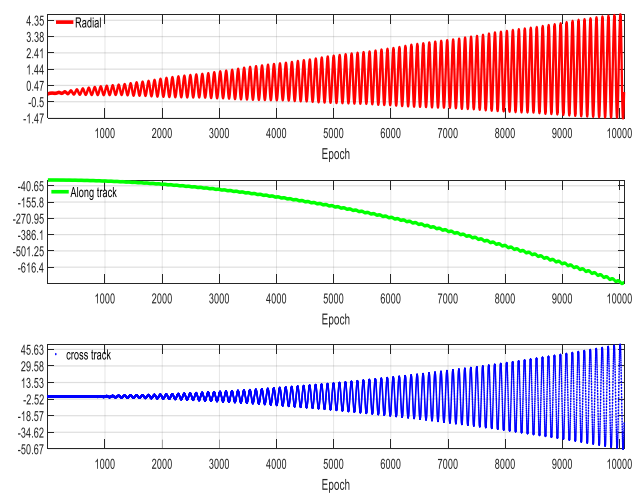

(b)

Figure 7. Orbital differences between the predicted orbits and the precise ones within 3 days (a), and 7 days (b), using the DTM87 atmospheric density model. 
Table 8. The statistics of orbit differences between the predicted orbits and the precise ones within 3 days and 7 days using the DTM87 atmospheric density model (m).

\begin{tabular}{cccccccc}
\hline & \multicolumn{2}{c}{ Radial } & \multicolumn{2}{c}{ Along Track } & \multicolumn{2}{c}{ Cross Track } & \multicolumn{2}{c}{ 3-D } \\
\cline { 2 - 8 } & STD & RMS & STD & RMS & STD & RMS & RMS \\
\hline 3 days & 0.502 & 0.616 & 41.653 & 62.332 & 2.982 & 2.983 & 62.406 \\
7 days & 1.272 & 1.506 & 219.122 & 330.629 & 16.202 & 16.202 & 331.029 \\
\hline
\end{tabular}

Figure 7 and Table 8 show that the orbital disagreement among the predicted orbits using DTM87 and the precise orbits for the 3-day arc was $0.616 \mathrm{~m}$ and the one for the 7-day arc was $1.506 \mathrm{~m}$ in radial direction. The accuracy was better than that from MSIS86, and worse than that from Jacchia 1971.

The above figures, tables, and analyses suggest that the predicted orbits using the Jacchia 1971 atmospheric density model had the best agreement with SSALTO orbits in the long time periods in three dimensions, and the accuracy of predicted orbits using the 3-day arc was significantly better than that using 7-day arc, which certified that the accuracy of predicted orbits will be decreased with the extension of time span.

\subsection{Analysis of Repeated Ground Track}

In order to assess the repeating ground track for HY-2A, the predicted ground trajectory of this mission for 28 days is shown in Figure 8.

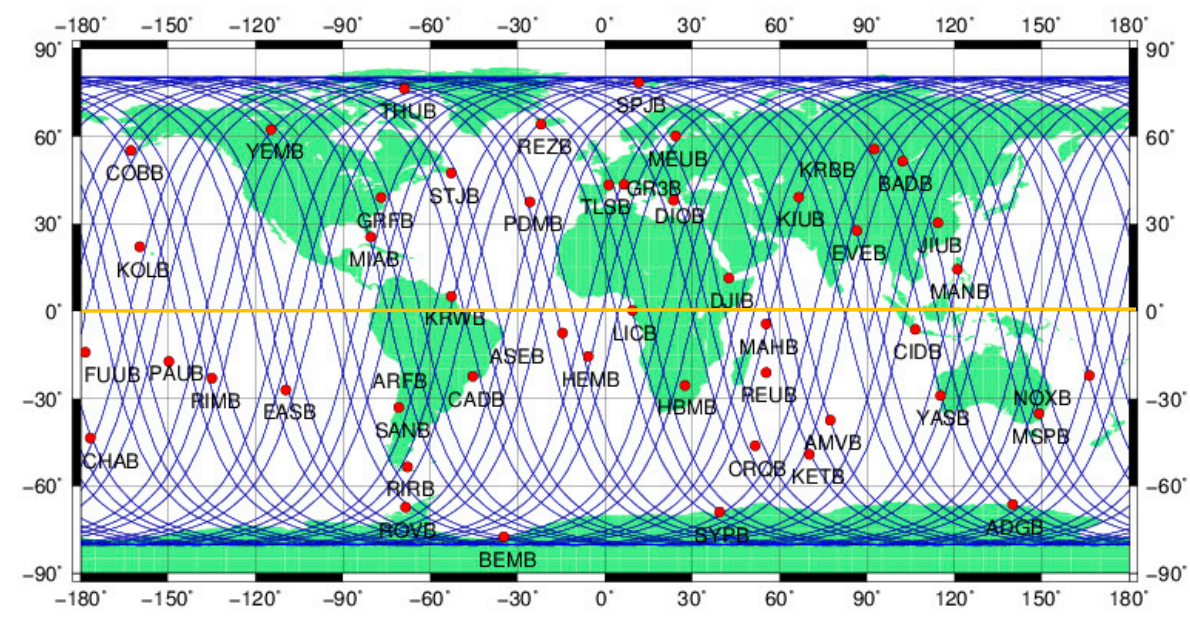

Figure 8. The ground trajectory predicted using Jacchia 1971 model for the HY-2A satellite for 28 days.

According to Equations (2) and (3), comparing the equatorial longitudinal distance between the equatorial crossing points of prediction trajectory and those of precise trajectory derived from SSALTO is an effective method of evaluating the accuracy of orbit prediction. To inspect the effect of our prediction strategy, we computed the ascending nodes and descending nodes with three atmospheric density models using the 3-day and 7-day arcs during 28 days, including two repeating cycles. At the same time, we also calculated the position of equatorial crossing points using precise orbits for 28 days. There are 256 nodes for these two successive periods. The equatorial distances of the successive ascending nodes and descending nodes between the predicted ground tracks using the three atmospheric density models and the precise ground track are plotted in Figures 9 and 10, and the statistics are summarized in Table 9. 


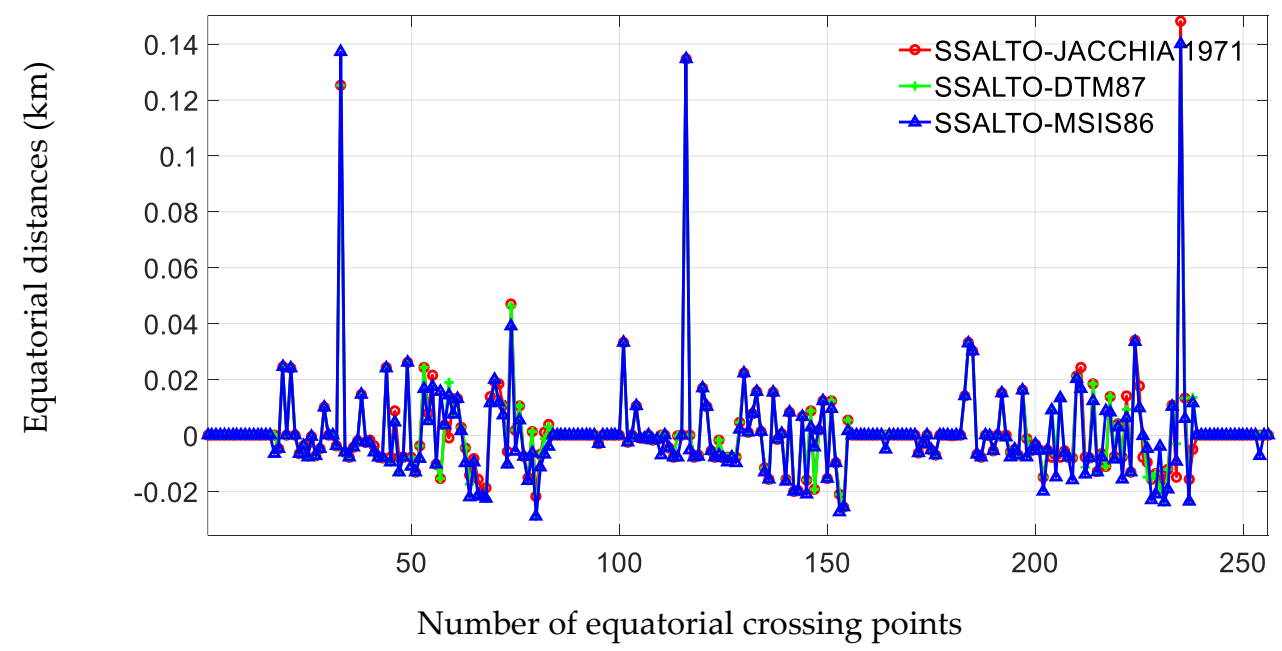

Figure 9. The equatorial distances of equatorial crossing points between the predicted trajectory with three atmospheric density models using the 3-day arc and precise trajectory during two successive periods of 28 days.

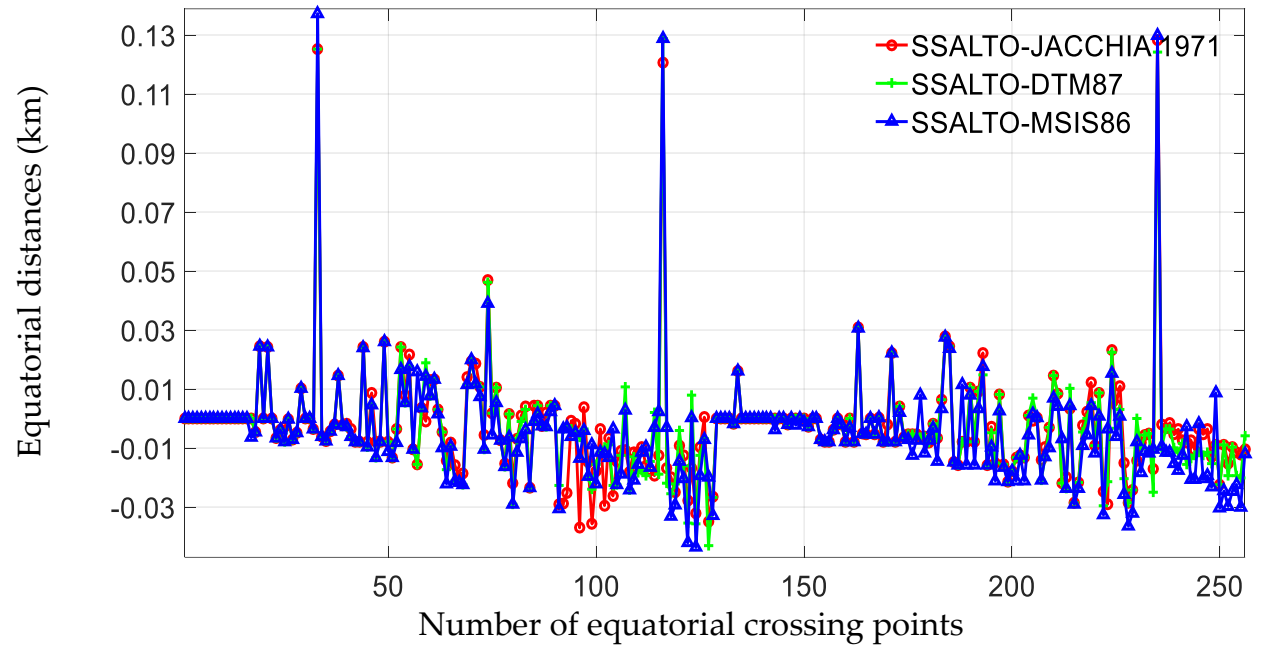

Figure 10. The equatorial distances of equatorial crossing points between the predicted trajectory with three atmospheric density models using 7-day arc and precise trajectory during two successive periods of 28 days.

Table 9. Statistics of the equatorial distances of equatorial crossing points between the trajectory predicted with three atmospheric density models using the 3-day and 7-day arcs and a precise trajectory during two successive periods of 28 days $(\mathrm{km})$.

\begin{tabular}{lllllll}
\hline & 3-Day Arc & & \multicolumn{5}{l}{ 7-Day Arc } \\
\hline & $\begin{array}{l}\text { SSALTO- } \\
\text { Jacchia 1971 }\end{array}$ & $\begin{array}{l}\text { SSALTO- } \\
\text { MMIS86 }\end{array}$ & $\begin{array}{l}\text { SSALTO- } \\
\text { DTM87 }\end{array}$ & $\begin{array}{l}\text { SSALTO- } \\
\text { Jacchia 1971 }\end{array}$ & $\begin{array}{l}\text { SSALTO- } \\
\text { MSIS86 }\end{array}$ & $\begin{array}{l}\text { SSALTO- } \\
\text { DTM87 }\end{array}$ \\
\hline MAX & 0.1380 & 0.1401 & 0.1381 & 0.1282 & 0.1372 & 0.1289 \\
MIN & -0.0257 & -0.029 & -0.0290 & -0.037 & -0.0437 & -0.043 \\
STD & 0.0171 & 0.0184 & 0.0180 & 0.0186 & 0.0196 & 0.0187 \\
RMS & 0.0171 & 0.0184 & 0.0180 & 0.0188 & 0.0200 & 0.0190 \\
\hline
\end{tabular}

Figures 9 and 10, and Table 9 indicate that the equatorial distances between the predicted orbits and precise orbits were enlarged a few meters with the extension of the prediction arc length. The RMS of equatorial distances using the 3-day arc length were about $0.0171 \mathrm{~km}, 0.0184 \mathrm{~km}$, and $0.0180 \mathrm{~km}$ using Jacchia 1971, MSIS86, and DTM87, respectively, and the RMSs using 7-day arc interval were 
$0.0188 \mathrm{~km}, 0.0200 \mathrm{~km}$, and $0.0190 \mathrm{~km}$ using Jacchia 1971, MSIS86, and DTM87, respectively, which show that the predicted trajectory with Jacchia 1971 had the best agreement with the SSALTO trajectory. Both tests indicated that the equatorial distances between the predicted trajectory and precise trajectory were smaller than $1.0 \mathrm{~km}$. The predicted orbits obtained using our strategy could meet the needs of HY-2A trajectory design. The Jacchia 1971 atmospheric model has a few advantages in the computation of equatorial distances of ground tracks between the predicted orbits and precise orbits.

According to Equations (2) and (3), comparing the equatorial distances of predicted orbits between successive repeating cycles is another effective method of validating the accuracy of predicted orbits, and this method can also test and verify the validity of the trajectory design and control. The repeating cycle period of the HY-2A satellite is about 14 days. Therefore, to compare the equatorial distances of ground tracks for HY-2A, we carried out orbital prediction for 28 days using the three atmospheric density models. The equatorial distance between the predicted orbits of successive periods using the 3-day arc and the 7-day arc are plotted in Figures 11 and 12, respectively, and the statistics are summarized in Table 10. To compare the predicted equatorial distances with those of the precise ones, we also computed the equatorial distances of the precise orbits, as shown in Figures 11 and 12, and statistics are listed in Table 10.

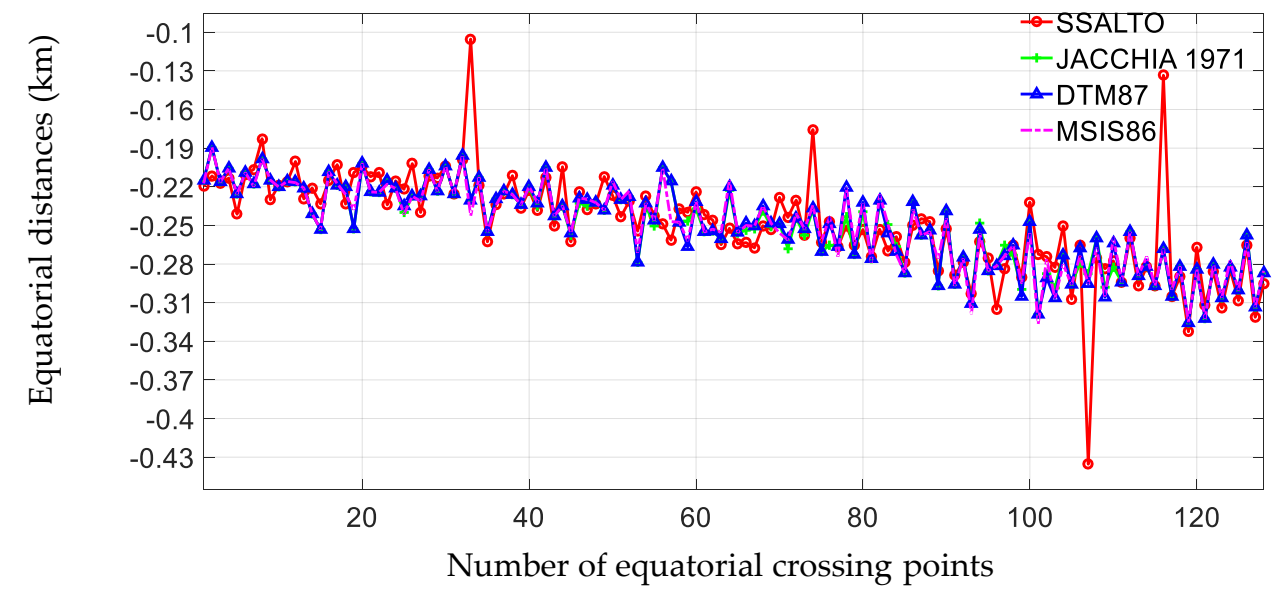

Figure 11. Equatorial distances of predicted and SSALTO ground tracks between two successive repeating cycles for the HY-2A satellite using 3-day arc.



Figure 12. Equatorial distances of predicted and SSALTO ground tracks between two successive repeating cycles for the HY-2A satellite using 7-day arc. 
Table 10. The statistics of disagreement of ground track between two successive repeating cycles for the HY-2A satellite using the 3-day arc and the 7-day arc (km).

\begin{tabular}{llllllll}
\hline & \multicolumn{3}{c}{ 3-day Arc } & \multicolumn{3}{c}{ 7-day Arc } \\
& SSALTO & $\begin{array}{l}\text { Jacchia } \\
\text { 1971 }\end{array}$ & MSIS86 & DTM87 & Jacchia & MSIS86 & DTM87 \\
\hline MAX & -0.1053 & -0.1894 & -0.1894 & -0.1894 & -0.1827 & -0.1827 & -0.1827 \\
MIN & -0.4354 & -0.3251 & -0.3266 & -0.3256 & -0.3112 & -0.3389 & -0.3308 \\
STD & 0.0395 & 0.0311 & 0.0315 & 0.0320 & 0.0290 & 0.0322 & 0.0352 \\
RMS & 0.2515 & 0.2519 & 0.2521 & 0.2521 & 0.2505 & 0.2535 & 0.2526 \\
\hline
\end{tabular}

From Figures 10 and 11, and Table 10, we found that the equatorial ground track distances between the successive periods were all better than $0.3 \mathrm{~km}$ using the three different atmospheric density models using the 3-day and 7-day arcs, and that of SSALTO was about $0.2515 \mathrm{~km}$ and better than that predicted using the three atmospheric density models. The RMSs of the equatorial ground track distance derived from Jacchia 1971 for 3-day and 7-day arcs were $0.2519 \mathrm{~km}$ and $0.2505 \mathrm{~km}$, respectively, and those from MSIS86 were $0.2521 \mathrm{~km}$ and $0.2535 \mathrm{~km}$, and those from DTM87 model were $0.2521 \mathrm{~km}$ and $0.2526 \mathrm{~km}$. We found that the accuracy of equatorial ground track distance of the 3-day arc was better than that of the 7-day arc for MSIS86 and DTM87, while that for Jacchia 1971 was somewhat different. The main reason is that coincidence of predicted results between the adjacent period derived from Jacchia 1971 for the 7-day arc was a little more stable than those for the 3-day arc judging from the value of STD. The above analyses show that the relatively optimal accuracy of equatorial distances for the 3-day and 7-day arcs were both derived from the Jacchia 1971 model, and the predicted orbits could meet the requirements of better than $1 \mathrm{~km}$. The analysis also showed that the three atmospheric models were all stable when predicting the orbit for HY-2A during the 28 days, and the trajectory controls for HY-2A were all effective.

In order to check the repeating ground track, we also computed the equatorial longitudinal separation between two successive ground tracks using SSALTO orbits for HY-2A, JASON-2, and JASON-3. JASON-2 and JASON-3 had a similar repeating cycle of about 10 days. The equatorial longitudinal separation of ground track between two successive periods for these three satellites are exhibited in Figure 13, and the statistics are summarized in Table 11.

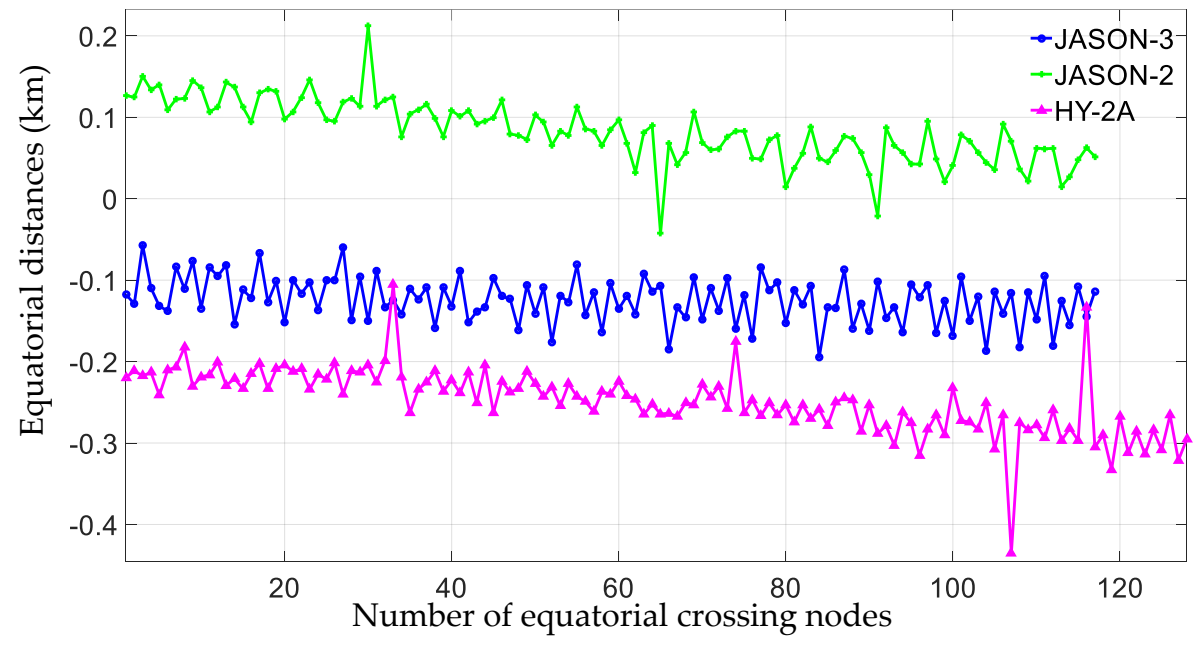

Figure 13. The equatorial longitudinal separation of ground track between two successive periods for HY-2A, JASON-2, and JASON-3 satellites. 
Table 11. The statistics of the equatorial longitudinal separation of ground track between two successive periods for HY-2A, JASON-2, and JASON-3 satellites.

\begin{tabular}{lllll}
\hline & MAX & MIN & STD & RMS \\
\hline JASON-3 & -0.0570 & -0.1946 & 0.0281 & 0.1276 \\
JASON-2 & 0.2122 & -0.0426 & 0.0379 & 0.0915 \\
HY-2A & -0.1053 & -0.4354 & 0.0395 & 0.2515 \\
\hline
\end{tabular}

Figure 13 and Table 11 exhibit that the RMS of the equatorial longitudinal separation for JASON-2 was $0.0915 \mathrm{~km}$, and the one for JASON-3 was $0.1276 \mathrm{~km}$. Both of them were better than that of HY-2A. As can be seen from Figure 12 and Table 11, JASON-3 had the most stable equatorial distances between successive periods among these three satellites, and JASON-2 had more stable equatorial distances than HY-2A did, which is demonstrated by STD in Table 11. Compared with JASON-3, there was space for HY-2A to improve the trajectory to maintain considerable stability and accuracy of equatorial distances between successive periods to improve the quality of application for altimetry data. One possible reason may be that the JASON's altitude of about $1336 \mathrm{~km}$ was higher than that of HY-2A of about $970 \mathrm{~km}$, which makes the JASON satellites receive less atmospheric resistance than HY-2A does. Another reason may be that the capability of trajectory control for JASON was better than that of HY-2A.

\section{Discussion}

The launch of the high-accuracy and high-resolution series altimetry satellite of the HY-2 is on schedule. To ensure the effectiveness of the application of data and provide guidance for satellite orbit maneuvering, orbit prediction and quality evaluation of altimetry satellites is essential. This work will provide an important reference for other altimetry satellite orbit predictions and can play a guiding role, especially for the precise orbit prediction for LEO navigation satellites to be launched in the future. Furthermore, this work will be helpful to the study of space weather involving the atmospheric density models.

To obtain precise orbit prediction for the HY-2A satellite, three classical atmospheric density models are adopted in this paper, and the difference between predicted orbit and precise orbit are compared and evaluated in short-term and long-term arcs. To test the ground track of the predicted orbit in the repetitive cycle, a method of interpolation special for spherical surface was introduced to calculate the intersection of the trajectory and the equator, and the equatorial distances between the predicted ground track and precise track and between predicted ground track of successive repeating cycles were analyzed in detail.

Previous studies have described the HY-2A orbit prediction $[7,8]$. These studies neither carry out pure dynamic orbit prediction from the initial orbital parameters nor compare the atmospheric density models. Therefore, it is difficult to judge which atmospheric density model is suitable for HY-2A and to test the perfection of the dynamic models. Wang et al. [7] needed to know the precise orbits throughout the forecast period and then fitted the satellite orbits. Zhu et al. [8] applied ARMA algorithms for orbit prediction, but this method could not reflect the physical mechanism of satellite orbits.

The accuracy evaluation methods of predicted orbits for an altimetry satellite are very important. The accuracy of the predicted orbits directly determines whether the satellite altimetry data analysis is correct or not, and whether the satellite is required to issue orbital maneuvers. Most of the research done compares the forecasting orbit with the precision orbit [7,8]. This paper not only compares with the precision orbit but also compares the ground track of predicted orbit, and it assesses the equatorial distance of the ground track between the successive repeating cycles.

The trajectory design and control of HY-2A should be further improved. As an HY-2 series satellite, HY-2B is one of the HY-2 series ocean dynamic satellites. HY-2B was launched on October 25, 2018, and will form a network with the subsequent HY-2C and HY-2D for maritime environmental 
monitoring. The research of this paper will provide a theoretical and methodological reference for the orbit prediction of these missions.

\section{Conclusions}

The main purpose of this article was to promote the optimal atmospheric density model to predict the orbit for the HY-2A satellite, thus providing theoretical and methodological reference for orbit prediction of other altimeter satellites, especially for the HY-2 series of satellites.

To validate the result of orbit prediction with three models for short-term and long-term time periods, we have predicted HY-2A orbits for $1 \mathrm{~h}, 2 \mathrm{~h}, 4 \mathrm{~h}, 8 \mathrm{~h}, 12 \mathrm{~h}, 24 \mathrm{~h}, 3$ days, and 7 days using Jacchia 1971, MSIS86, and DTM87 atmospheric density models. Compared with the precise orbits, the smallest radial RMSs values could reach $0.016 \mathrm{~m}, 0.176 \mathrm{~m}, 0.573 \mathrm{~m}$, and $1.421 \mathrm{~m}$ for $1 \mathrm{~h}, 1$ day, 3 days, and 7 days, respectively, which were all obtained from the Jacchia 1971 atmospheric density model. To assess the predicted orbit, equatorial distances in the longitude direction have also been computed. The smallest equatorial distances between the predicted ground track and the precise ones were $0.0171 \mathrm{~km}$ and $0.0188 \mathrm{~km}$ for 3-day and 7-day arcs, respectively, using Jacchia 1971, and the smallest ones between the predicted ground track of successive repeating cycles were $0.2519 \mathrm{~km}$ for 3-day arcs and $0.2505 \mathrm{~km}$ for 7-day arcs using Jacchia 1971. The studies indicated that using Jacchia $1971 \mathrm{can}$ achieve the best predicted orbits, followed by DTM87, and the worst was using MSIS86. The largest RMS value difference was between Jacchia 1971 and MSIS86, followed by a smaller difference between Jacchia 1971 and DTM87, and the least one was between DTM87 and MSIS86. The main reasons include the following aspects. First, the MSIS-class models had not included drag measurements and satellite-borne accelerometer data, which makes it deficient to be applied to orbital prediction. Second, the MSIS-class models are derived from the DTM, which causes a small difference between the RMS values of predicted results derived from two models. Third, Jacchia 1971 is mostly based on drag data obtained by observing the orbital motion of numerous satellites.

To evaluate the effect of trajectory control for the HY-2A satellite, we also compared the equatorial distances of the ground track with the successive periods for HY-2A, JASON-2, and JASON-3, and the result showed that the equatorial distances of ground track between the successive periods for HY-2A are about $0.25 \mathrm{~km}$ and are obviously worse than those of JASON-2 and JASON-3. One possible reason may be that the altitude of HY-2A is lower than that of JASON, which makes HY-2A suffer larger atmospheric drag than JASON-2 and JASON-3 do. Another reason may be that the capability of trajectory control for JASON is better than that of HY-2A.

From the test results, the following conclusions can be drawn. First, the predicted orbits with Jacchia 1971 have the best agreement with the precise orbits, and as the arc extended, the orbital disagreement became larger and larger, and the disagreement of Jacchia 1971 was the smallest among the three models. Second, the ability to obtain high-precision predicted orbits for the HY-2A satellite depended primarily on our well-established orbital dynamics model. Third, the interpolation method especially used for a spherical surface could effectively calculate the position of the interpolation point on the spherical surface for the ground track, thus providing an effective way for the evaluation of the repeated trajectory of the ground track. Fourth, the trajectory design and control of HY-2A need to be improved to better meet the requirements of the altimetry data.

However, for long-term trend analysis, greater effort should be put into researching the recently derived atmospheric density models, which will provide important practical application value in trajectory design and coverage evaluation for the following HY-2 series altimetry satellites.

Author Contributions: Q.K., F.G., and J.G. designed the experiment. Q.K. analyzed the data. Q.K. and F.G. wrote the paper. L.H., L.Z., and Y.S. proposed amendments. All authors have reviewed the manuscript.

Acknowledgments: The authors are grateful to the editors and anonymous reviewers for their helpful comments, which led a significant improvement in this paper. This work was supported by the National Natural Science Foundation of China (Grant No. 41704015, 41774001), the Shandong Natural Science Foundation of China (Grant No. ZR2017MD003, ZR2017MD032), a Project of Shandong Province Higher Educational Science and Technology 
Program (Grant No. J17KA077), SDUST Research Fund, and Joint Innovative Center for Safe and Effective Mining Technology and Equipment of Coal Resources.

Conflicts of Interest: The authors declare no conflict of interest.

\section{References}

1. Meng, X.Q. Research and Application of Satellite Orbit Prediction Method Based on Osculating Kepler Element. Master's Thesis, University of Chinese Academy of Sciences, Beijing, China, 2017. (In Chinese)

2. Circi, C.; Ortore, E.; Bunkheila, F. Satellite constellations in sliding ground track orbits. Aerosp. Sci. Technol. 2014, 39, 395-402. [CrossRef]

3. Wu, X.Y. Research and Application on Remote Sensing Satellite High Precision to Cover the Distributed Algorithm Based on the Geometric Topology. Master's Thesis, Henan University, Zhengzhou, China, 2016. (In Chinese)

4. Mur, T.J.M.; Springer, T.; Bar-Sever, Y. Orbit predictions and rapid products. In Proceedings of the 1998 Analysis Centre Workshop of the International GPS Service for Geodynamics (IGS), Darmstadt, Germany, 9-11 February 1998.

5. Romay, M.M.; Lainez, M.D. Generation of precise long-term orbit and clock prediction products for A-GNSS. In Proceedings of the International Technical Meeting of the Satellite Division of the Institute of Navigation, Nashville, TN, USA, 17-21 September 2012.

6. Polle, B.; Sidorov, D.; Marie, S.; Dach, R.; Gonzalez, F. Orbit/SRP modelling for long term prediction. In Proceedings of the IGS Workshop 2017, Paris, France, 3-7 July 2017.

7. Wang, Y.; Zhong, S.; Wang, H.; Ou, J. Precision analysis of LEO satellite orbit prediction. Acta Geod. Cartogr. Sin. 2016, 45, 1035-1041. (In Chinese)

8. Zhu, Y.H.; Chao, X.; Cai, C.L. Method and analysis of medium and long term satellite orbit prediction based on satellite broadcast ephemeris parameters. Int. J. Future Gener. Commun. Netw. 2015, 8, 187-196. [CrossRef]

9. Tang, J.; Liu, L.; Cheng, H.; Hu, S.; Duan, J. Long-term orbit prediction for TianGong-1 spacecraft using the mean atmosphere model. Adv. Space Res. 2015, 55, 1432-1444. [CrossRef]

10. Hartikainen, J.; Seppanen, M.; Sarkka, S. State-space inference for non-linear latent force models with application to satellite orbit prediction. In Proceedings of the 29th International Conference on Machine Learning, Edinburgh, Scotland, UK, 26 June-1 July 2012; pp. 1206-1215.

11. Jacchia, L.G. Revised Static Models of the Thermosphere and Exosphere with Empirical Temperature Profiles; Special Report in Smithsonian Astrophysical Observatory; SAO: Cambridge, MA, USA, 1971.

12. Hedin, A.E. MSIS-86 thermospheric model. J. Geophys. Res. 1987, 92, 4649-4662. [CrossRef]

13. Gaposchkin, E.M.; Coster, A.J. Analysis of satellite drag. Lincoln Lab. J. 1988, 1, 203-224.

14. Martin, T. MicroCosm System Description; Van Martin Systems Inc.: Green Bay, WI, USA, 2011.

15. Montenbruck, O.; Gill, E. Satellite Orbits: Models Methods Applications, 2nd ed.; Springer: Heidelberg, Germany, 2001; pp. 91-102. ISBN 3-540-67280-X.

16. Vallado, D.A.; Finkleman, D. A critical assessment of satellite drag and atmospheric density modeling. Acta Astronaut. 2014, 95, 141-165. [CrossRef]

17. Fattig, E.; McLaughlin, C.; Lechtenberg, T. Comparison of Density Estimation for CHAMP and GRACE Satellites. In Proceedings of the AIAA/AAS Astrodynamics Specialist Conference, Toronto, ON, Canada, 2-5 August 2010.

18. Thaheer, A.S.M.; Ismail, N.A. Orbit Design and Lifetime Analysis of MYSat: A 1U CubeSat for Electron-density Measurement. In Proceedings of the 4th NatGrad Conference, Uniten, Malaysia, 26-27 April 2017.

19. Berger, C.; Biancale, R.; Ill, M.; Barlier, F. Improvement of the empirical thermospheric model DTM: DTM-94-A comparative review of various temporal variations and prospects in space geodesy applications. J. Geodesy 1998, 72, 161-178. [CrossRef]

20. Knudsen, P.; Brovelli, M. Collinear and cross-over adjustment of Geosat ERM and Seasat altimeter data in the Mediterranean Sea. Surv. Geophys. 1993, 14, 449-459. [CrossRef]

21. Scharroo, R.; Visser, P. Precise orbit determination and gravity field improvement for the ERS satellites. J. Geophys. Res. Oceans 1998, 103, 8113-8127. [CrossRef] 
22. Montenbruck, O.; Gill, E. Satellite Tracking and Observation Models, Satellite Orbits, 1st ed.; Springer: Heidelberg, Germany, 2000; pp. 87-98. ISBN 978-3-642-58351-3.

23. Balmino, G.; Barriot, J.P. Numerical integration techniques revisited. Manuscr. Geod. 1990, 15, 1-10.

24. Pavlis, N.K.; Holmes, S.A.; Kenyon, S.C.; Factor, J.K. The development and evaluation of the Earth Gravitational Model 2008 (EGM2008). J. Geophys. Res. Solid Earth 2012, 117, 1-38. [CrossRef]

25. Standish, E.M. JPL Planetary and Lunar Ephemerides, DE405/LE405; Jet Propulsion Laboratory, InterOffice Memorandum (IOM): Pasadena, CA, USA, 1998; pp. 1-18.

26. Petit, G.; Luzum, B. IERS Conventions (2010); Bureau International des Poids et Mesures: Sèvres, France, 2010.

27. Lyard, F.; Lefevre, F.; Letellier, T.; Francis, O. Modeling the global ocean tides: Modern insights from FES2004. Ocean Dyn. 2006, 56, 394-415. [CrossRef]

28. McCarthy, D.D.; Petit, G. Equations of Motion for an Artificial Earth Satellite; IERS Technical Note 32; IERS Conventions: Paris, France, 2003.

29. Rim, H.J. TOPEX Orbit Determination Using GPS Tracking System. Ph.D. Thesis, University of Texas at Austin, Austin, TX, USA, 1992.

30. Knocke, P.C.; Ries, J.C.; Tapley, B.D. Earth radiation pressure effects on satellites. In Proceedings of the AIAA/AAS Astrodynamics Conference, Guidance, Navigation, and Control and Co-Located Conferences, Reston, VA, USA, 15-17 August 1998; pp. 577-587.

31. Goad, C.C.; Goodman, L. A modified Hopfield tropospheric refraction correction model. In Proceedings of the American Geophysical Union Annual Fall Meeting, San Francisco, CA, USA, 12-17 December 1974.

32. Gao, F.; Peng, B.; Zhang, Y.; Evariste, N.H.; Liu, J.; Wang, X.; Zhong, M.; Lin, M.; Wang, N.; Chen, R.; et al. Analysis of HY2A precise orbit determination using DORIS. Adv. Space Res. 2015, 55, 1394-1404. [CrossRef]

(C) 2018 by the authors. Licensee MDPI, Basel, Switzerland. This article is an open access article distributed under the terms and conditions of the Creative Commons Attribution (CC BY) license (http:/ / creativecommons.org/licenses/by/4.0/). 